\title{
Event-based State-Space Model Predictive Control of a renewable hydrogen-based microgrid for office power demand profiles ${ }^{\star}$
}

\author{
M. Castilla ${ }^{\mathrm{a}, *}$, C. Bordons ${ }^{\mathrm{b}}$, A. Visioli $^{\mathrm{c}}$ \\ ${ }^{a}$ Centro Mixto CIESOL, ceiA3, Universidad de Almería. Ctra. Sacramento s/n. 04120, \\ Almería, Spain \\ ${ }^{b}$ Universidad de Sevilla. Camino de los Descubrimientos, s/n. 41092, Seville, Spain \\ ${ }^{c}$ University of Brescia. via Branze 38. 25123, Brescia, Italy
}

\begin{abstract}
This paper focuses on the design and implementation of an event-based control architecture to manage a renewable-based microgrid. This microgrid has renewable-energy generation and a hybrid energy storage system that uses electricity and hydrogen. The main load of the microgrid is the energy demand of an office. The primary control objective is to satisfy the load demand using the available renewable generation and stored energy while reducing the amount of energy purchased from the Utility Power Grid (UPG). To do that, the control architecture, which has been defined within an event framework, makes use of a set of state-space model predictive controllers which are selected as a function of a variable sampling period. To evaluate the performance of the proposed control architecture, simulation tests for a typical day of summer as well as an analytical study is performed. Furthermore, a comparison of the obtained results with a classic state-space model predictive control approach is also included. The obtained results show that the use of the event-based control architecture allows a significant reduction of the number of changes in the control action at
\end{abstract}

\footnotetext{
${ }^{\star}$ This work has been funded by the research project CONFIGURA (DPI201678338-R) from the Spanish Ministry of Economy and Competitiveness and AGERAR (0076_AGERAR_6_E) founded by the European Commission.

${ }^{*}$ Corresponding author

Email addresses: mcastilla@ual.es (M. Castilla), bordons@us.es (C. Bordons), antonio.visioli@unibs.it (A. Visioli)
}

Preprint submitted to Journal of Power Sources

October 9, 2019 
the expense of an acceptable deterioration of set-point tracking for a microgrid with several types of electrochemical storage.

Keywords: Event-based control, Model Predictive Control, microgrids, thermal comfort, energy efficiency, co-simulation

\section{Introduction}

These days, a transition from centralized and fossil-fuels based energy systems to distributed renewable-energy based ones is taking place mainly due to the fact that non-renewable energy sources are more and more exhausted [1]. Hence, it is necessary to develop new control algorithms able to manage the effects related to the intermittence and distribution of energy, as well as new demand profiles. For this reason, the use of microgrids has spread in recent years. A microgrid can be defined as a group of generators, loads and energy storage systems that can be managed in both connected to grid or islanded operating modes with the principal aim of providing electricity in a reliable way [2]. In that way, a microgrid can be used to guarantee Quality of Service for local loads just as hospitals, shopping centers, office buildings, etc.

Nevertheless, as it is pointed out in [3], the development of microgrid control algorithms is a difficult task which presents several problems and important challenges. Specifically, [3] focuses on optimal management of microgrids. Moreover, it is possible to find in literature many other works related to energy management of a microgrid which make use of different approaches, such as, heuristic algorithms [4], stochastic control [5, 6, neural-network and fuzzy logic techniques [7. Besides, Model Predictive Control (MPC) is one of the most used techniques since it allows the integration of both generation and load predictions and energy price estimations. For instance, in [8] optimal controllers for distributed renewable generation networks and batteries storage are presented. Likewise [9, 10, illustrates the use of centralized MPC techniques. In [11] a review of different types of control approaches applied to microgrids (such as rule-based, model predictive control and occupancy based model predictive 
control for buildings) is performed.

On the other hand, the use of event-based control approaches presents several advantages, such as the computation of new control signals only when an event is detected or the reduction in the use of resources [12. Besides, these approaches are very beneficial when they are applied to distributed systems, since they can decrease the information exchange among the elements which compose the system [13. For these reasons, in the last years the use of eventbased techniques has spread widely and they have been applied to a huge variety of systems. For example, to control Heating, Ventilation and Air-Conditioning (HVAC) systems in buildings [14, 6, distributed solar collector fields [15], pH level in photobioreactors [16, etc. However, up to our knowledge, there are not many works which have applied event-based control to manage a microgrid.

Sometimes it is required to consider specific variables like the connection status (connected/disconnected) or changes in the behaviour of the equipment during the normal operation (for example different charging/discharging efficiency coefficients for a batteries bank) within the control problem. For this reason, it is necessary to develop and analyse new configuration, modelling and control techniques. Therefore, the main objective of this paper is the development of a control approach within the framework of hybrid MPC which allows the efficient management of a renewable-energy based microgrid with two types of electrochemical storage: batteries and hydrogen (fuel cell and electrolyzer). Moreover, the developed strategy has considered some deterioration of storage systems criteria to provide a larger durability. To do that, the use of eventbased MPC control is ideal since it maintains all the individual advantages of both MPC and sampling techniques based on events. In more detail, these characteristics of an event-based MPC have been used to establish a trade-off between the performance of the control system (accuracy) and the necessary effort (resources used by the control strategy). In this way, event-based control systems can reduce the use of resources (by the application of new control signals only if it is required), and thus, also the economical costs at the expense of an insignificant deterioration of the control system performance. 
A control approach has been developed in order to manage an existing laboratory-scale renewable-energy based microgrid located at the University of Seville [17. The power supported by this microgrid is similar to the one demanded by an office room, see Table 1 . The principal goals of the proposed control system, from a performance point of view, are to satisfy realistic load profiles for an office-building, to maintain the energy storage levels within established security limit and to minimize the amount of energy exchanged with the Utility Power Grid (UPG). Additionally, the degradation of the storage devices (battery, electrolyzer and fuel cell) is taken into account; as analysed in [18, this is closely related to the changes in the control actions, so a control strategy that avoids frequent and sudden changes in the use of storage devices can improve their lifetime. More in detail, it has been supposed that the microgrid should satisfy the energy demanded by an office-room considering its thermal comfort specifications and the physical constraints of the microgrid itself. Hence, a building has been simulated and controlled by means of co-simulation techniques and the total energy amount demanded by a room of this building has been quantified taking into account not only the energy demanded by the HVAC system but also, the energy demanded by artificial lighting and electrical appliances. Henceforth, to manage the renewable-energy based microgrid, an event-based state-space MPC control has been designed and implemented. In this case, two different types of events have been defined: threshold and time-based events. The effectiveness of the proposed control architecture has been analysed by means of several simulation tests for a typical summer day in Seville. In addition, the obtained results have been compared with a classic state-space MPC approach. The obtained results are promising since they demonstrate that it is possible to reduce the number of actuations around a $65 \%$, at the expense of an acceptable deterioration of the performance and an increment of the energy exchanged with the UPG less than $1.35 \mathrm{kWh}$ for a whole day of operation for the selected solution. Further, even if less important in this application, the computing time is reduced up to $70 \%$.

The structure of the rest of the paper is organized as follows: Section 2 
describes the methodology used to obtain realistic energy demand profiles for an office-room considering thermal comfort constraints. Section 3, describes the renewable-energy microgrid. Section 4 performs a detailed explanation of the event-based state-space control architecture presented in this paper. Section 5 introduces the optimization procedure followed to select the main parameters of the event-based strategy. Section 6 presents the simulation results obtained for a typical summer day in Seville. Finally, Section 7 summarises main conclusions and future works.

\section{Energy demand: Thermal comfort control of an office-room}

In this paper, it has been supposed that the renewable-energy based microgrid is used to satisfy the energy demanded by an office-room. Therefore, obtaining realistic energy consumption profiles is a cornerstone, and thus, it is necessary to determine the energy required by its users in order to develop their daily activities, taking into consideration thermal comfort constraints.

Besides, for the purpose of assuring a comfortable situation for the users of a room from a thermal point of view, the use of adequate control systems is required [19. Moreover, to design and implement appropriate control strategies, it is fundamental to have a building model able to capture its dynamic behaviour. In this paper, an office building and a room-level thermal comfort controller have been simulated by means of co-simulation techniques. Co-simulation can be defined as the integration of various simulation tools at run-time coupling [20, 21]. More specifically, the EnergyPlus engine [22] has been used to simulate the dynamic behaviour of the building whereas the thermal comfort controller has been designed using Matlab. Furthermore, it is required to establish a communication at simulation time between EnergyPlus and Matlab, and thus, the Building Control Virtual TestBed (BCVTB) tool has been utilised [23].

In more detail, one of the buildings which have been included as an example of how to use BCVTB tool has been selected and modified in order to be considered as an office building. It occupies an estimated total surface equal to $310 \mathrm{~m}^{2}$ 
and comprises two floors and a basement. At each floor, two thermal zones which face South and North have been considered, see figure 1(a). However, to evaluate the performance of the event-based state-space MPC architecture presented in this paper, the south zone of the first floor $(Z S F 1)$ has been selected as a characteristic office-room due to the dimensions of the renewable-energy based microgrid. Furthermore, the following assumptions have been considered in order to simulate the building:

- The maximum occupation rate of the selected room has been established to 10 people. Moreover, it has been fixed that the first five workers arrive at 7 a.m. Afterwards, at 8 a.m. three more workers arrive to their workplace. The occupation rate of the room is fixed to its maximum value from 10 a.m. to 1 p.m. and from 3 p.m. to 5 p.m. From 1 p.m. to 3 p.m. and from 5 p.m. to 6 p.m. the occupation rate has been established to five people. Finally, the last two workers abandon the room at 7 p.m., see $1(b)$.

- The building is located in the city of Seville. So, hourly weather data for Seville obtained from 22] has been used. This dataset contains International Weather for Energy Calculations (IWEC) data originated based on almost 20 years of data originally archived at the U.S. National Climatic Data Centre and supplemented by solar radiation calculated as a function of earth-sun geometry and cloud amount information [24]. As an example, main outdoor environmental variables for a typical summer day can be observed in figure 1 (c).

- The HVAC systems are operated from 6 a.m. to 7 p.m..

As it can be observed in figure 1(c), Seville has hot summers with a maximum mean outdoor temperature approximately equal to $36^{\circ} \mathrm{C}$. Therefore, to reach a comfortable thermal sensation inside buildings the use of active strategies such as HVAC systems, able to control indoor climate, is required. At this point, it is important to mention that EnergyPlus allows the control of the 


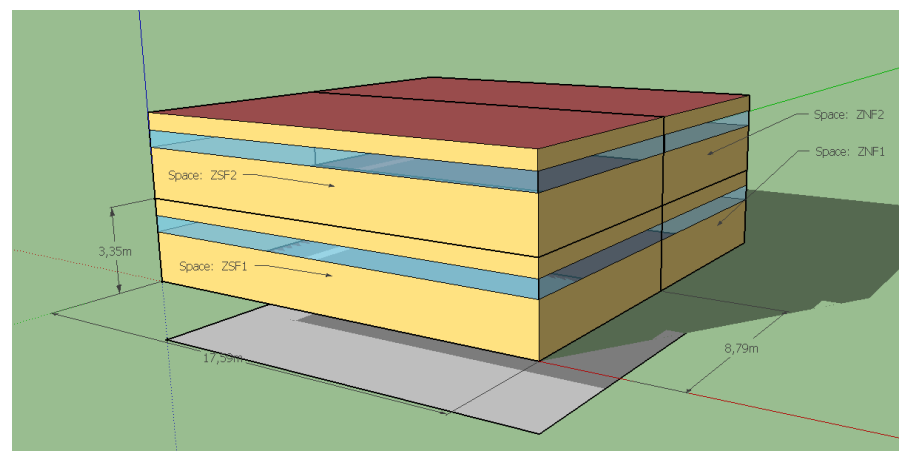

a) Simulated office building: 3D overview

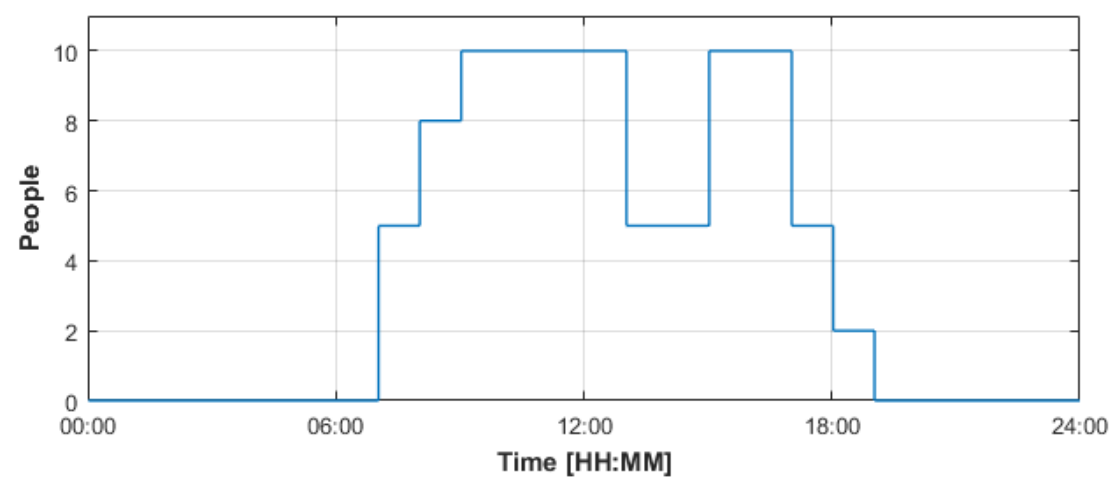

b) Profile of people inside the room

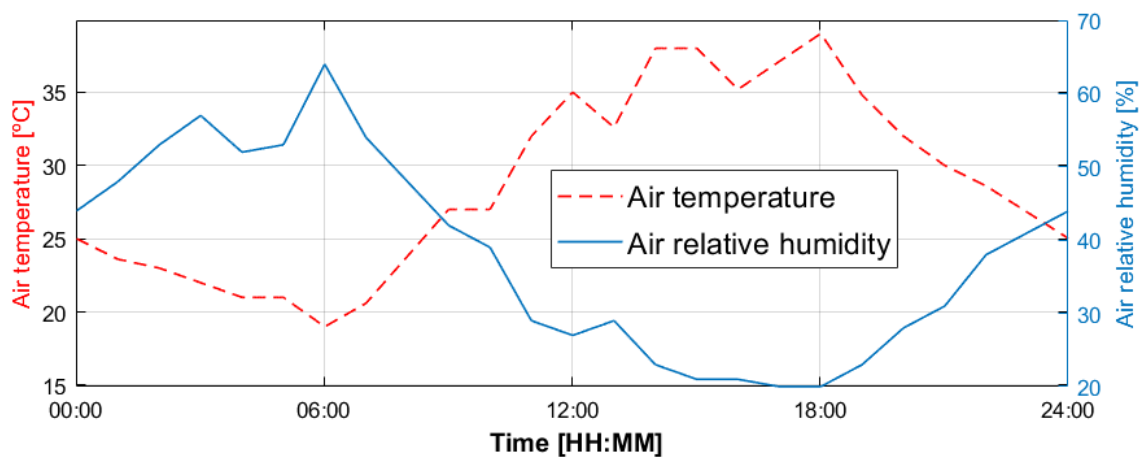

c) Outdoor conditions for summer in Seville

Figure 1: Principal characteristics of the building simulated with EnergyPlus 
indoor environment through zone level control algorithms by modifying, in an appropriate way, the set-points defined for these zones. Those algorithms can be defined as a function of a thermostat, of the operative temperature or the Predicted Mean Vote (PMV) index [25]. Therefore, the designed control algorithm in Matlab should estimate the adequate set-points based on the selected zone control algorithm, that is, indoor air temperature, operative temperature or PMV index, respectively. In this paper, a zone control based on the PMV index has been performed. This index allows the prediction of the mean response about thermal sensation of a large group of people exposed to particular thermal conditions for a certain amount of time. Concretely, the steps that have been followed to develop a thermal comfort controller for an office-room using co-simulation techniques can be summarized as follows:

- An office building has been configured and simulated in EnergyPlus. It will be considered as a room simulator from thermal comfort control point of view.

- A characteristic room has been selected $(Z S F 1)$ and a model of it has been obtained by means of classical identification techniques [26]. To do that, a Pseudo-Random Binary Signal (PRBS) has been designed in Matlab and it has been applied to the EnergyPlus building model using BCVTB tool. The main goal was to capture enough data of the selected room to allow the identification of a model of the room.

- Afterwards, a simple control signal has been designed and implemented in Matlab in order to maintain the PMV index within the limits established by international standards. To do that, the model identified beforehand has been used. Moreover, it should be taken into consideration that this controller is subject to several disturbances such as outdoor air temperature and room occupation. Simulation results of the thermal comfort controller can be observed in figure 2 .

- Finally, once that the room simulator (office building model developed in 
EnergyPlus) and the thermal comfort controller (Matlab) have been designed and implemented, a control architecture which allows the thermal comfort sensation to be maintained into the selected room has been implemented using co-simulation techniques. To do that, EnergyPlus and Matlab will simulate the building and the thermal comfort controller respectively. Moreover, they will exchange the necessary information for a correct operation using BCVTB interface at run-time coupling.

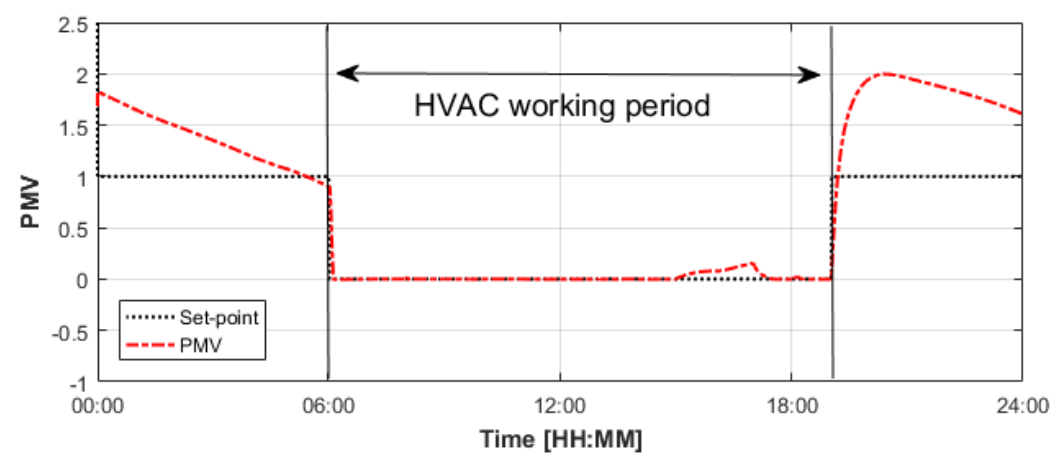

Figure 2: Results of the thermal comfort controller within the selected room

A more detailed description of the methodology followed to identify the room model and to implement the thermal comfort control system has not been included, since it is out of the scope of the work presented in this paper. Several works and results related to this topic can be found in 19 .

Finally, the energy demanded by this room to satisfy users' necessities related to their quotidian activities and thermal comfort has been obtained from EnergyPlus simulation engine. Specifically, the energy consumed by artificial lighting and electrical appliances has been considered and moreover, the energy used by the HVAC system to maintain a thermal comfort sensation has also been taken into consideration. These energy demands have been configured to depend on the number of people and the type of lights and electrical appliances inside the room. Therefore, it has been supposed that there is a computer with similar characteristics for each person. Data related to lighting and electrical 
appliances energy consumption for office-rooms have been obtained from [27]. Finally, a summary of the energy demanded by this room can be observed in figure 3. This profile of energy demanded will be used from hereafter.

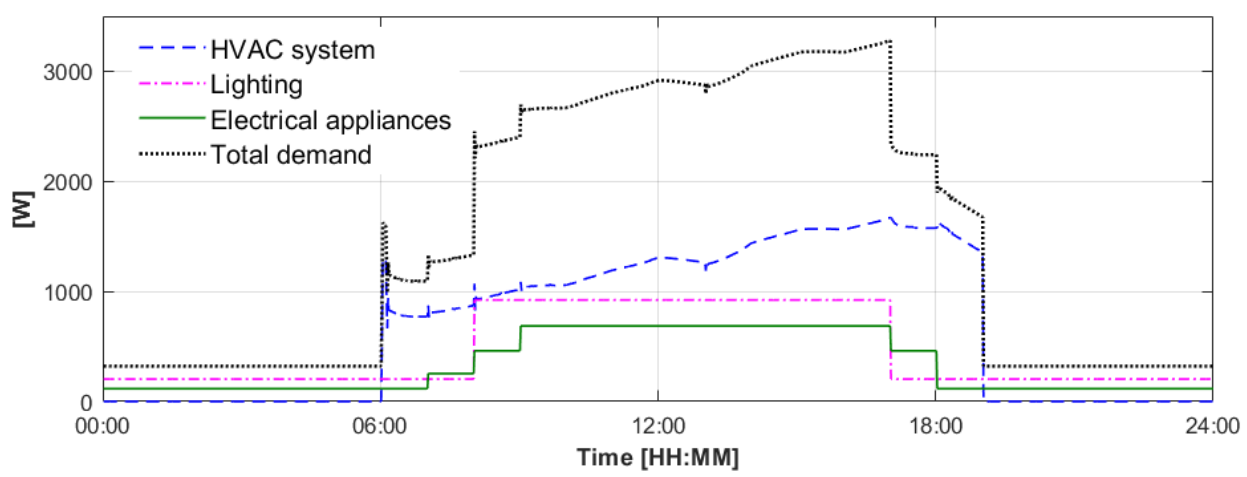

Figure 3: Energy demanded by the office-room

\section{Renewable-energy based microgrid}

The plant which is being analysed in this paper is an experimental renewableenergy based microgrid located at the University of Seville (Spain). It is utilised to test control approaches applied to energy management. The microgrid makes use of hydrogen storage and renewable energy sources [9, 17, 28]. Figure 4 shows a picture of the experimental microgrid, also known as Hylab, as well as its principal components.

In general, under normal operation conditions of the microgrid, the energy demanded is not usually equal to the production. Hence, surplus energy from renewable sources can be kept in batteries or utilised to generate hydrogen by means of electrolysis. In more detail, the hydrogen generated is stored in the metal hydride tank. Thus, if the power from renewable energy sources is not enough, the fuel cell can use hydrogen in order to supply the energy demanded. Besides, the microgrid is connected to the main grid which allows energy sale and purchase. The availability of hybrid storage (hydrogen and electricity) allows the development of operation strategies on two different time scales: hydrogen 


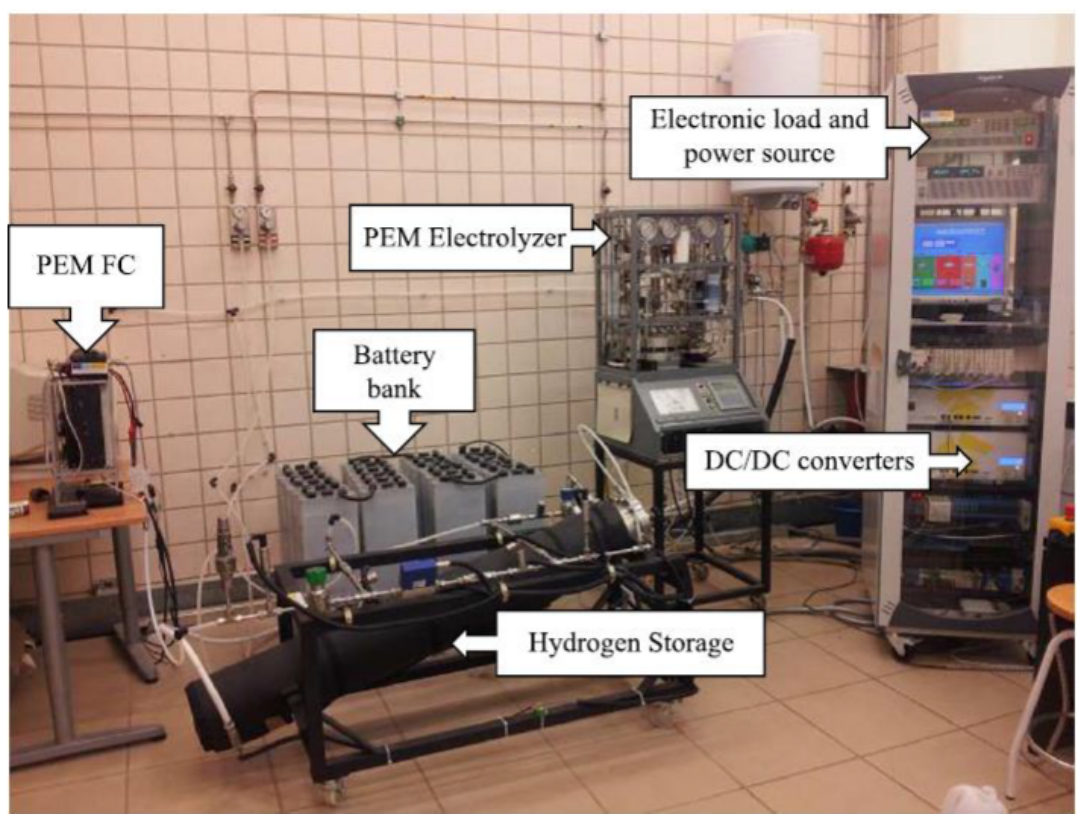

Figure 4: Hylab microgrid.

can be used to supplement bigger oscillations while the batteries bank is responsible of absorbing/providing small quantities of energy on fast transients. To replicate the dynamic behaviour of renewable energy sources, the microgrid uses a programmable power supply which can simulate a photovoltaic field and/or a wind turbine. In addition, it also incorporates an electronic load to replicate several demand profiles, a batteries bank and a complete hydrogen storage system which is composed of a metal hydride tank used to keep hydrogen, a PEM (Proton Exchange Membrane) fuel cell to produce electricity and a PEM electrolyzer to generate hydrogen. The technical characteristics of the main components of the microgrid can be observed in table 1 .

The components of the microgrid are connected to a $48 \mathrm{~V} D C$ current bus using adequate power electronics. In addition, the control system has a dedicated central control based on a programmable logic controller (PLC). This device is specifically responsible for the necessary calculations and for determining the basic control actions. The electrolyzer and fuel cell are managed through power 
converters and Controller Area Network (CAN) bus communications. On the contrary, the electronic load and the power supply are controlled in analogue mode. Generally, control systems which make use of a model of the system are high in computational demands, and thus, real-time implementation using commercial PLCs is to some extent problematic. Hence, in order to deal with this problem, MPC control actions are determined using Simulink Real-Time software on a computer installed in the laboratory. Afterwards, the computer sends the determined control actions to the PLC using MATLAB OPC library. The MPC control system receives the plant outputs to estimate the optimal sequence of control signals.

To allow the DC bus to transfer power, two DC/DC converters related to the fuel cell and the electrolyzer have been used. On the contrary, the batteries bank is connected directly to the DC bus. Therefore, to simplify the topology, the bus voltage is held by the batteries bank. This implementation is very common in DC microgrids since it allows reducing costs and increasing reliability mainly due to the fact that any unbalance in the system is absorbed by the batteries bank [29]. Moreover, the converters related to the demand and renewable energy sources are electronically emulated. A complete description of the microgrid design and a full characterization of each subsystem can be found in [17.

Table 1: Microgrid Units.

\begin{tabular}{ll}
\hline Equipment & Nominal Value \\
\hline Programmable Power Supply & $6 \mathrm{~kW}$ \\
Electronic Load & $2.5 \mathrm{~kW}$ \\
Electrolyzer & $0.23 \mathrm{Nm}^{3} \mathrm{~h}^{-1}$ at $1 \mathrm{~kW}$ \\
Metal Hydride Tank & $7 \mathrm{Nm}^{3}$ at $5 \mathrm{bar}$ \\
Fuel Cell & $1.5 \mathrm{~kW}$ at $20 \mathrm{Nl}$ \\
Battery Bank & $C 120=367 \mathrm{Ah}$ \\
\hline
\end{tabular}




\subsection{Linear model}

A key aspect to design and implement an adequate control architecture for the renewable-energy based microgrid is to have an available model. This model must be able to precisely represent its dynamic behaviour. Therefore, assuming that the efficiency of the battery is the same for charge and discharge, the following energy balance equations determine the increment in the level of energy in the storage units:

$$
\begin{aligned}
& S O C(t+1)=S O C(t)-\frac{\eta_{b a t} T_{s}}{C_{\max }} P_{b a t}(t) \\
& L O H(t+1)=L O H(t)+\frac{\eta_{e z} T_{s}}{V_{\max }} P_{e z}(t)-\frac{T_{s}}{\eta_{f c} V_{\max }} P_{f c}(t)
\end{aligned}
$$

where $S O C$ and $L O H$ are the State Of Charge of the batteries bank and the Level of Hydrogen of the hydrogen storage tank in [\%] respectively, $\eta$ is the conversion efficiency of each storage unit, $C_{\max }$ is the maximum energy capacity of the battery and $V_{\max }$ is the maximum volume of $H_{2}$ that can be stored in the tanks. Then, $P_{b a t}$ is the power supplied by the battery, $P_{e z}$ is the power consumed by the electrolyzer and $P_{f_{c}}$ is the electric power generated by the fuel cell. Notice that the battery is directly connected to the DC bus and absorbs the unbalance, so $P_{b a t}$ must compensate the rest of powers in the DC bus:

$$
P_{b a t}(t)=P_{d e m}(t)+P_{e z}(t)-P_{f c}(t)-P_{g r i d}(t)-P_{r e n}(t)
$$

So, defining $\omega(t)=P_{\text {ren }}(t)-P_{\text {dem }}(t)$ as the measurable disturbance, that is, the existing difference between the power $P_{r e n}$ originated by renewable energy sources and the power $P_{d e m}$ demanded, the storage equations are:

$$
\begin{aligned}
& S O C(t+1)=S O C(t)-\frac{\eta_{b a t} T_{s}}{C_{\max }}\left(P_{e z}(t)-P_{f c}(t)-P_{\text {grid }}(t)-\omega(t)\right) \\
& L O H(t+1)=L O H(t)+\frac{\eta_{e z} T_{s}}{V_{\max }} P_{e z}(t)-\frac{T_{s}}{\eta_{f c} V_{\max }} P_{f c}(t)
\end{aligned}
$$

For the sake of simplicity, the powers of the hydrogen path are grouped in one variable $P_{H_{2}}=P_{f c}-P_{e z}$, assuming that the efficiencies are similar in 
both ways. The values of the conversion from charging power to electrical and hydrogen storage are obtained performing several experiments on the plant. A series of 168 simulations were performed for different operating points: SOC and $\mathrm{LOH}$ between $10 \%$ and $90 \%$ and charging and discharging powers between 500 and $1750 \mathrm{~W}$. Finally, a discrete-time linear state-space model of the plant around an operating point $\left(u^{*}, x^{*}\right)$ has been obtained by means of identification techniques:

$$
x(k+1)=x(k)+\left[\begin{array}{cc}
0.0936 & 0.0936 \\
-0.3397 & 0
\end{array}\right] u(k)+\left[\begin{array}{c}
0.0936 \\
0
\end{array}\right] \omega(k)
$$

where $x=[S O C ; L O H]$ represents the states and $u=\left[P_{H 2} ; P_{\text {grid }}\right]$ in $[k W]$ is the vector of the two manipulated inputs variables: the power applied to the hydrogen path (electrolyzer, hydrogen storage tank and fuel cell) and the power traded between the UPG and the microgrid. Finally, this model has been discretized with a sampling period of $T_{s}=10 \mathrm{~s}$. Moreover, the following assumptions have been considered:

- It has been supposed that the fuel cell and the electrolyzer cannot operate simultaneously. Therefore, if $P_{H 2}$ is lower than 0 , it is associated with the power of the electrolyzer $\left(P_{e z}\right)$. On the other side, if $P_{H 2}$ is positive, it represents the power $P_{f c}$ obtained from the fuel cell.

- A negative value of $P_{\text {grid }}$ stands for the power which is exported to the UPG. On the contrary, a value of $P_{\text {grid }}$ greater than zero represents the power imported from the UPG to the microgrid.

\section{Event-based State-Space MPC for a renewable-energy based mi- crogrid}

In this section, the event-based state-space MPC architecture presented in this paper is introduced. Concretely, it is based on the algorithm proposed in [12. The main difference lies in the MPC algorithm selected to be integrated 
within the event-based architecture. More in detail, the control architecture presented in [12] is based on the Generalized Predictive Control (GPC) algorithm, while, in this paper, a state-space formulation has been used. The use of a state-space formulation simplifies the problem because it is not necessary to perform signal sampling and re-sampling techniques since state-space MPC only needs to know the state of the controlled variables and control signals in the current time instant and, in our case, these variables can be measured. Moreover, to select the parameters for the event-based architecture a multiobjective optimization problem has been solved, see Section 5 .

In this paper, a modified version of this algorithm has been applied to control the experimental laboratory microgrid described in Section 3 . Hence, it is a Multiple-Input-Multiple-Output (MIMO) control problem. More specifically, the microgrid has two outputs: $S O C$ and $L O H$ which are controlled by means of two manipulated variables: $P_{H 2}$ and $P_{\text {grid }}$. Besides, this process is subject to a disturbance, $\omega$, that is, the difference between the power $P_{r e n}$ generated by renewable energy sources, and the power $P_{d e m}$ demanded by an office-room.

\subsection{Control architecture}

Generally, an event-based control strategy can be considered as a combination of feedforward and feedback actions. Furthermore, it can also be classified as a type of hybrid control since the system will run in open loop between events [30]. An event based control architecture is composed of three main elements: an event-detector unit, a controller unit and the process unit, see figure 5 In more detail, each one of these elements is responsible for the following tasks:

- Process unit: This module represents the controlled process, that is, the experimental microgrid. To do that, a simulation based on a non-linear model of it has been used [28].

- Event-detector unit: This element receives the outputs of the controlled process $(S O C$ and $L O H)$ and analyses if an event has happened. In this 
case, it looks for two types of events: time events and threshold ones. Afterwards, the occurrence of an event and the exact time instant it has happened are sent to the controller unit.

- Controller unit: This unit is composed of a set of state-space MPC (SSMPC) controllers and an event classification sub-unit. Moreover, it is responsible for obtaining new control signals $\left(P_{H_{2}}\right.$ and $\left.P_{\text {grid }}\right)$ when an event is detected. Concretely, the event classification module analyses the received event and selects the most appropriate SSMPC controller as a function of time in which the new event is detected. Afterwards, the selected SSMPC controller recalculates control signals by solving the MPC problem defined in Section 4.3 ,

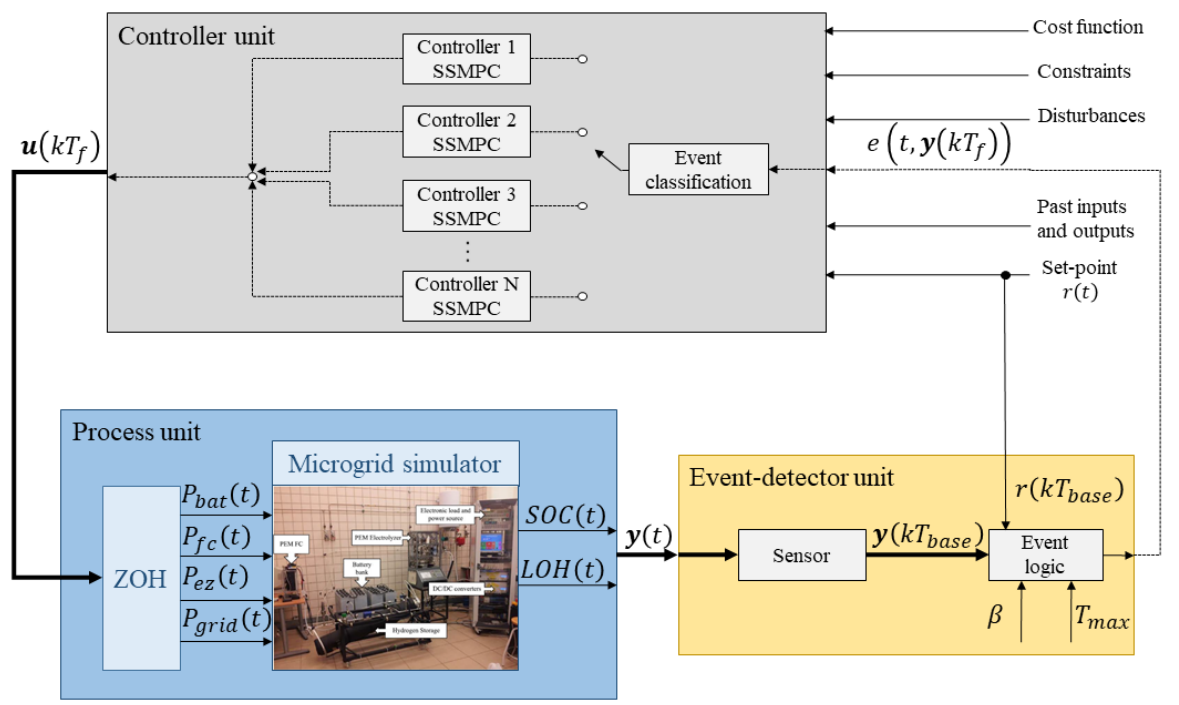

Figure 5: Event-based state-space MPC control architecture.

In order to operate correctly the event-based state-space MPC control strategy, it is necessary to define some concepts and constraints related to different sampling times and the occurrence of events:

- The outputs of the controlled process, that is, $S O C$ and $L O H$ are provided by the microgrid simulator. These variables are measured by means of a 
Sensor module and sampled through a constant sample time denoted as $T_{\text {base. }}$.

- Afterwards, the event-logic module analyses at each $T_{b a s e}$ time instant the process outputs in order to identify the occurrence of a new event. Specifically, two types of events have been defined: threshold based ones, which make use of a parameter denoted $\beta$; and time-based events, that depend on $T_{\max }$. More information about the event-logic module can be found in Section 4.2 .

- Finally, control signals $\left(P_{H 2}, P_{\text {grid }}\right)$ are calculated and applied to the microgrid as a function of a variable sampling period $T_{f}$, which depends on the occurrence of an event. $T_{f}$ must be a multiple of $T_{\text {base }}$ and below a maximum sampling period $T_{\max }=n_{\max } \cdot T_{\text {base }}$. The selection of $T_{\max }$ is an important cornerstone in this type of controllers since it will influence their performance. Hence, it is necessary to define a set of state-space MPC controllers based on the variable sampling period $T_{f}$. This group of controllers makes use of the state-space model given by (6) discretized with different sampling periods $\left(T_{f} \in\left\{T_{\text {base }}, 2 \cdot T_{\text {base }}, \ldots T_{\max }\right\}\right)$.

In the following subsections, a detailed explanation of the main elements which compose the proposed control architecture has been included.

\subsection{Event-logic module}

In an event-based controller, control signals are not estimated in a synchronous mode, but are calculated when a new event is detected. Therefore, the sampling period of the controlled system is regulated by the event-logic module. More specifically, it is responsible for verifying some conditions over the process in order to detect possible events, and whenever necessary, to update the sampling period of the controlled system and the state-space MPC controller used.

As discussed before, in this paper two types of conditions have been defined as events. Hence, when one of those conditions is detected, the event-logic 
module generates a new event which is transmitted to the controller unit together with the main process variables necessary to generate a new set of control signals.

In particular, the first condition is associated with a threshold event applied to the outputs of the controlled process. In other words, a new event is generated when the existing difference between one of the outputs of the process $(S O C$ or $L O H)$ and their associated set-point values is greater than a fixed tolerance $\beta$, see $(7)$. This type of conditions is known as level crossing sampling technique [31], and it can be formally expressed as,

$$
|w(t)-y(t)|>\beta
$$

where the output of the system $y(t)$ coincides with the state $x(t)$, that is, $y(t)=x(t)=[S O C(t) ; L O H(t)]$.

On the contrary, the second condition is related to a time event which is used for stability and performance improvement. Specifically, this condition generates a new event when the time between two consecutive events is greater that a maximum established time, that is, $T_{\max }$, see (8). Formally, it is

$$
t-t_{e_{i}} \geq T_{\max }
$$

where, $t$ is the current time-instant and $t_{e_{i}}$ represents the time-instant in which the last event was detected.

Therefore, to detect a new event, at each period $T_{\text {base }}$ the previous mentioned conditions are checked, and this will originate a variable sampling period denoted as $T_{f}$. As it can be inferred from (7) and (8), the selection of $\beta$ and $T_{\max }$ is a critical issue which should be addressed in an appropriate way.

\subsection{State-Space Model Predictive Control}

As it was discussed previously, the controller unit within the event-based control architecture is composed of a set of state-space MPC controllers. In this section, the design of a classic state-space MPC controller which has been 
discretized using a certain sample time $T_{f}$ is explained. MPC is distinguished by the explicit use of a model of the controlled system in order to predict its state along a prediction horizon $N_{p}$ [32. Specifically, every $T_{f}$ time period, a sequence of control signals $\left\{u(t), \cdots, u\left(t+N_{p}-1\right)\right\}$ is obtained by optimizing a cost function, see (9). Then, the first component of this sequence is applied to the microgrid at time instant $t$ while the others are dismissed. Subsequently, the complete procedure is performed again at next sampling time $(t+1)$ using more updated information. The cost function is expressed as

$$
\begin{aligned}
J\left(N_{p}, N_{u}\right) & =\sum_{j=1}^{N_{p}-1}\left[\hat{x}(t+j \mid t)-x_{r e f}(t+j)\right]^{T} \delta\left[\hat{x}(t+j \mid t)-x_{r e f}(t+j)\right] \\
& +\sum_{j=1}^{N_{u}-1}[\Delta u(t+j \mid t)]^{T} \lambda[\Delta u(t+j \mid t)] \\
& +\sum_{j=1}^{N_{u}-1}[u(t+j \mid t)]^{T} \gamma[u(t+j \mid t)]
\end{aligned}
$$

where, $\hat{x}(t+j \mid t)$ represents an optimal $j$ step-ahead prediction of the system output estimated at sampling time $t+j$ with the information available at sampling time $t, N_{p}$ and $N_{u}$ are the prediction and control horizons, respectively, and $\delta, \lambda$ and $\gamma$ are weighting matrices used to balance the set-point tracking, the control increment and the control effort, respectively. Finally, $x_{r e f}(t+j)$ is the desired set-point for the states. Besides, it is worthwhile to mention that it has been considered that all the signals have a sampling period $T_{f}\left(t=k T_{f}, k=1, \cdots, \infty\right)$. In this paper, these values have been established as $\delta=\left[\begin{array}{lll}0.01 & 0 ; 0 & 0.01\end{array}\right], \lambda=\left[\begin{array}{lll}50 & 0 ; 0 & 1000\end{array}\right]$ and $\gamma=\left[\begin{array}{lll}10 & 0 ; 0 & 300\end{array}\right]$. These values have been selected to provide a higher priority to the use of the battery bank over the hydrogen path and, moreover, to reduce the exchange of energy with the UPG. Besides, set-point tracking weighting matrix, $\delta$, has been chosen not to guarantee a perfect set-point tracking but to maintain $S O C$ and $L O H$ within an established security limits that allow the reduction of the number of events, and thus, the number of actuations. Furthermore, the prediction 
and control horizons have been established to $N_{p}=5$ and $N_{u}=2$, respectively. Finally, the references values for $S O C$ and $L O H$ have been established to $x_{r e f}=\left[\begin{array}{ll}60 \% & 45 \%\end{array}\right]^{T}$, respectively since it has been considered to be the nominal operating point because it represents a compromise between operation flexibility and guaranteed operation autonomy. For this reason, the model used within the SSMPC has been linearized around this operation point.

In addition, at this point it is necessary to highlight that, although it is possible to find in literature other works which do not consider set-point tracking within storage systems [33, 34, in this paper it has been decided to include it as a not very representative term of the cost function with the purpose of not depleting storage systems.

Therefore, the state-space MPC controller is responsible for estimating the future control signals sequence by minimizing the cost function given by (9). To do that, it is required to obtain the optimal prediction of $x(t+j)$ for $j \in\left\{0,1, \cdots, N_{p}-1\right\}$ using the state-space model of the microgrid, see (6). The following assumptions have been considered in order to calculate the optimal prediction of $x(t+j)$. Firstly, the state-space model given by $(6)$ has been rewritten according to 10 where $A$ and $C$ are identity matrices of an appropriate size.

$$
\begin{aligned}
x(t+1) & =A x(t)+B u(t)+E \omega(t) \\
y(t) & =C x(t)
\end{aligned}
$$

As it was commented in Section 3 the system is subject to a state disturbance, $\omega(t)=P_{n e t}$, and thus, it is necessary to deal with it in an adequate way. To do that, the overall process model has been augmented to include the disturbance, following the formulation proposed by [35]. Although it would be ideal to include the disturbance forecast within the control problem, as it is not available, the disturbance has been considered constant over the entire prediction horizon, so that the augmented model results in 


$$
\begin{aligned}
\tilde{x}(t+1) & =A_{d} \tilde{x}(t)+B_{d} u(t) \\
y(t) & =C_{d} \tilde{x}(t)
\end{aligned}
$$

where,

$$
\begin{aligned}
\tilde{x}(t+1) & =\left[\begin{array}{c}
x(t+1) \\
\omega(t+1)
\end{array}\right] ; \quad A_{d}=\left[\begin{array}{cc}
A & E \\
0 & I
\end{array}\right] ; \\
B_{d} & =\left[\begin{array}{c}
B \\
0
\end{array}\right] ; \quad C_{d}=\left[\begin{array}{ll}
C & 0
\end{array}\right]
\end{aligned}
$$

Secondly, the state-space controller has been implemented using an incremental formulation of the state space model, that is, considering the control increment $\Delta u(t)$ instead of the control signal $u(t)$, where $\Delta u(t)=u(t)-$ $u(t-1)$ [32. Hence, defining a new state which depends of the previous augmented one $x_{f}=\left[\begin{array}{lll}\tilde{x}(t) & u(t-1)\end{array}\right]^{T}=\left[\begin{array}{lll}x(t) & \omega(t) & u(t-1)\end{array}\right]^{T}$, the incremental model takes the general form given by

$$
\begin{aligned}
x_{f}(t+1) & =M x_{f}(t)+N \Delta u(t) \\
y(t) & =Q x_{f}(t)
\end{aligned}
$$

where,

$$
M=\left[\begin{array}{cc}
A_{d} & B_{d} \\
0 & I
\end{array}\right] ; \quad N=\left[\begin{array}{c}
B_{d} \\
I
\end{array}\right] ; \quad Q=\left[\begin{array}{cc}
C_{d} & 0
\end{array}\right]
$$

that is,

$$
M=\left[\begin{array}{ccccc}
1 & 0 & 0.0936 & 0.0936 & 0.0936 \\
0 & 1 & 0 & -0.3397 & 0 \\
0 & 0 & 1 & 0 & 0 \\
0 & 0 & 0 & 1 & 0 \\
0 & 0 & 0 & 0 & 1
\end{array}\right] ; \quad N=\left[\begin{array}{cc}
0.0936 & 0.0936 \\
-0.3397 & 0 \\
0 & 0 \\
1 & 0 \\
0 & 1
\end{array}\right]
$$




$$
Q=\left[\begin{array}{lllll}
1 & 0 & 0 & 0 & 0 \\
0 & 1 & 0 & 0 & 0
\end{array}\right]
$$

Therefore, taking into consideration the issues mentioned above, the output of the model for sample time $t+j$, assuming that the value of states and future control increments are known, can be estimated recursively as

$$
y(t+j)=Q M^{j} x_{f}(t)+\sum_{i=0}^{N-1} Q M^{N-i-1} N \Delta u(t+i)
$$

In addition, this equation can be expressed in vectorial form just as: $\mathbf{y}=$ $\mathbf{F} \hat{x}(t)+\mathbf{H} \boldsymbol{\Delta} \mathbf{u}$, where $\mathbf{F}=\left[\begin{array}{llll}Q M & Q M^{2} & \cdots & Q M^{N_{p}}\end{array}\right]^{T} ; \mathbf{H}$ represents a block lower triangular matrix with its non-zero elements equal to $\mathbf{H}_{i j}=Q M^{i-j} N$ and $\boldsymbol{\Delta} \mathbf{u}=\left[\begin{array}{llll}\Delta u(t) & \Delta u(t+1) & \cdots & \Delta u\left(t+N_{p}-1\right)\end{array}\right]^{T}$ symbolises a sequence of future control increments. Hence, it can be also inferred that the output of the system depends on its current state and on a sequence of future control increments, which is the decision variable that have to be estimated.

The sequence of control increments $\boldsymbol{\Delta} \mathbf{u}$ is obtained by minimizing the cost function given by (9). Moreover, considering the recursive procedure to estimate the process output in its vectorial form, the cost function equation can be rewritten as:

$$
\begin{aligned}
J\left(N_{p}, N_{u}\right) & =\left(\mathbf{H} \boldsymbol{\Delta} \mathbf{u}+\mathbf{F} \hat{x}(t)-x_{r e f}\right)^{T} \delta\left(\mathbf{H} \boldsymbol{\Delta} \mathbf{u}+\mathbf{F} \hat{x}(t)-x_{r e f}\right) \\
& +\boldsymbol{\Delta} \mathbf{u}^{T} \lambda \boldsymbol{\Delta} \mathbf{u}+\mathbf{u}^{T} \gamma \mathbf{u}
\end{aligned}
$$

Finally, if the system is not subject to any constraint, it is possible to obtain an analytical solution from the previous equation. Nevertheless, in this case several constraints have been defined, and thus, a quadratic programming problem should be solved using the previously defined quadratic cost function with linear inequality and equality constraints [32]. In particular, several hard constraints have been included to ensure an adequate performance of the microgrid. In other words, they act as physical limitations of the connection for 
the grid operation and the hydrogen path by applying limitations to the control inputs variables and their increments:

$$
\begin{aligned}
-0.9 k W & \leq P_{H 2}(t) \leq 0.9 k W \\
-2.5 k W & \leq P_{\text {grid }}(t) \leq 2.5 k W \\
-0.9 k W s^{-1} & \leq \Delta P_{H 2}(t) \leq 0.9 k W s^{-1} \\
-2.5 k W s^{-1} & \leq \Delta P_{\text {grid }}(t) \leq 2.5 k W s^{-1}
\end{aligned}
$$

Furthermore, several constraints concerning the state variables, SOC and $L O H$, have been included with the main purpose of physically limiting the batteries bank and the level of hydrogen within the storage tank:

$$
\begin{aligned}
& 40 \% \leq S O C \leq 90 \% \\
& 10 \% \leq L O H \leq 90 \%
\end{aligned}
$$

Besides, it is worth emphasizing that the power of the batteries bank can be obtained from (3). Finally, The State-Space Model Predictive Control is summarized in Algorithm 1.

\section{Optimal selection of $T_{\max }$ and $\beta$ parameters}

As it was commented before, the choice of $T_{\max }$ and $\beta$ is a critical issue since it influences the performance of the obtained results. The main goal of the eventbased control architecture presented in this paper is to reduce the number of actuations without deteriorating too much the system's performance. Hence, it is a multiobjetive optimization problem.

A multiobjective optimization problem can be defined as the search for a vector of decision variables able to satisfy certain constraints and, at the same time, to optimize a vector of objective functions [36]. Therefore, a multiobjective problem is characterized by having two or more competing objectives (in conflict) which should be simultaneously minimized (or maximized) satisfying 
\begin{tabular}{l}
$\overline{\text { Algorithm } 1 \text { State-Space MPC problem }}$ \\
\hline Define the simulation time and current simulation time: $t_{\text {sim }}$ and $k=0$. \\
Define the initial conditions of manipulated inputs, outputs and disturbances.
\end{tabular}

while $k \leq t_{\text {sim }}$ do

1. Measure the current states $(\mathbf{x})$, manipulated inputs $(\mathbf{u})$, outputs $(\mathbf{y})$ and disturbances $(\omega)$.

2. Determine a new set of control signals along the control horizon, $\mathbf{u}=$ $\left[u(k \mid k), u(k+i \mid k), \ldots, u\left(k+N_{u}-1 \mid k\right)\right]$, by solving the quadratic problem defined by:

$$
\begin{aligned}
\min J\left(N_{p}, N_{u}\right) & =\quad \sum_{j=0}^{N_{p}-1} \delta\left[\hat{x}(k+j \mid k)-x_{\text {ref }}(k+j)\right]^{2} \\
& +\quad \sum_{j=0}^{N_{u}-1} \lambda[\Delta u(k+j \mid k)]^{2}+\sum_{j=0}^{N_{u}-1} \gamma[u(k+j \mid k)]^{2} \\
\text { s.t. } & \\
x(k+1) & =A x(k)+B u(k)+E \omega(k) \\
P_{\text {dem }}(k) & =P_{H 2}(k)+P_{\text {bat }}(k)+P_{\text {grid }}(k)+P_{\text {ren }}(k) \\
-0.9 k W & \leq P_{H 2}(k+j) \leq 0.9 k W \\
-2.5 k W & \leq P_{\text {grid }}(k+j) \leq 2.5 k W \\
-0.9 k W s^{-1} & \leq \Delta P_{H 2}(k+j) \leq 0.9 k W s^{-1} \\
-2.5 k W s^{-1} & \leq \Delta P_{\text {grid }}(k+j) \leq 2.5 k W s^{-1} \\
\forall j & =0 \ldots N_{u}-1
\end{aligned}
$$

3. The control signal $u(k \mid k)$ is applied to the process whilst the other control signals calculated in the previous step are discarded.

4. $\mathrm{k}=\mathrm{k}+1$;

\section{end while}


several constraints [37. The solution to this type of problems is not unique. In other words, it is not feasible to find an ideal solution able to optimize all the objective functions. Nevertheless, there is a possibility of determining a set of non-dominated solutions known as optimal solutions 37. which satisfy some selected criteria. More specifically, this choice is performed by using some decision techniques, such as, fixing the limit values of the decision variables.

A cornerstone within multiobjective optimization techniques is the definition of the problem. In fact, it is necessary to specify the objective functions in an appropriate way, that is, by determining which variables compose the objective functions and the existing relations among them and the decision variables in a clear and concise way.

In this case, there are two decision variables, $T_{\max }$ and $\beta$, which should optimize two different objectives: i) the number of events; and ii) the performance of the system by minimizing cost function evaluation, which indirectly optimizes $S O C$ and $L O H$ set-point tracking and the exchange of energy with the UPG. To do that, 132 simulations have been performed considering several values of $T_{\max }$ and $\beta$. In particular, it has been fixed that $\beta \in\{10 \%, 12 \%, \cdots, 30 \%\}$, $T_{\text {base }}=10 \mathrm{~s}$, and thus, $T_{\max } \in\{10 \mathrm{~s}, 20 \mathrm{~s}, \cdots, 120 \mathrm{~s}\}$. A summary of these simulations is depicted in figure 6. It shows the existing relationships between different combinations of $T_{\max }$ and $\beta$ parameters with the cost function evaluation (top) and the number of events (bottom). It can be seen from figure 6(top) that as $T_{\max }$ and $\beta$ parameters increase the performance of the system generally deteriorates quickly, and thus, better solutions for this objective are obtained for the smallest $T_{\max }$ and $\beta$ parameters. On the other hand, the minimum number of events is achieved with the maximum values of $T_{\max }$ and $\beta$ parameters while, with $T_{\max }=10 \mathrm{~s}$ and $\beta=1 \%$ a total number of events around 8000 has been reached. Therefore, both objectives are opposite regarding $T_{\max }$ and $\beta$ parameters.

Hence, a set of optimal solutions has been generated from the previous graphs, see figure 7. Concretely, it has been considered that a solution, that is a combination of $T_{\max }$ and $\beta$ parameters, belongs to the set of optimal solutions 

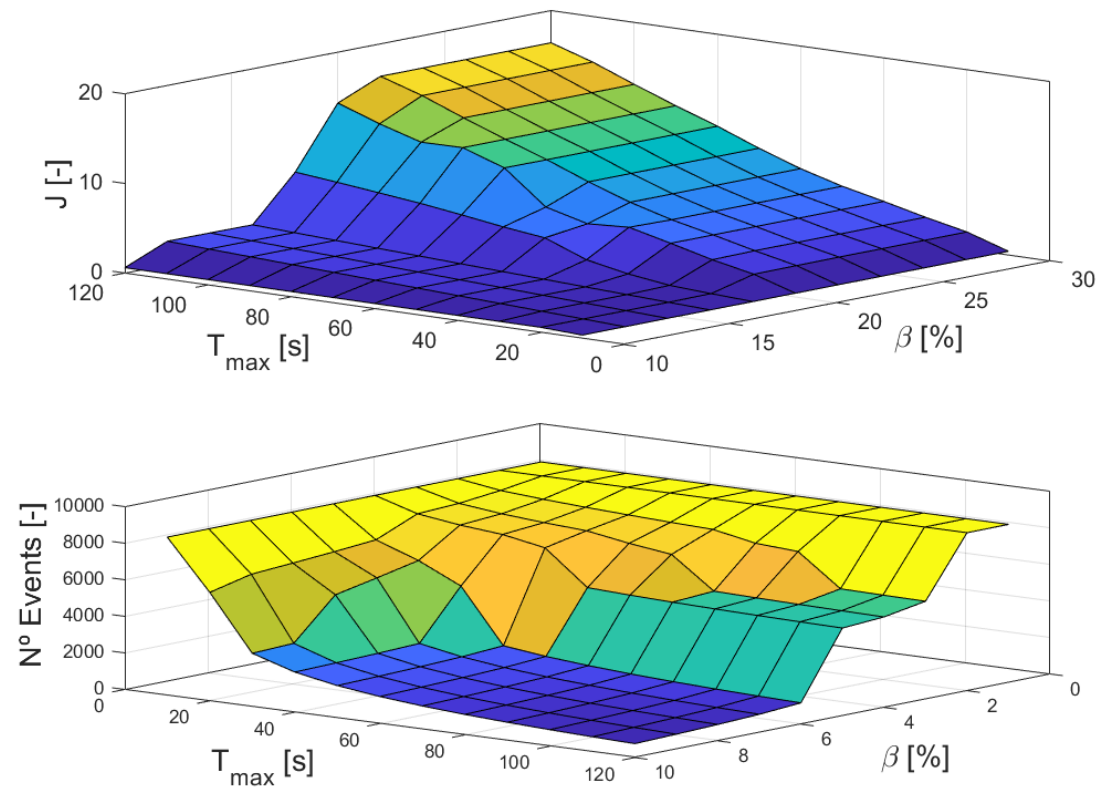

Figure 6: Variation of $J$ (top) and the number of events (bottom) as a function of $T_{\max }$ and $\beta$ parameters

if the performance of the system is lower than 9.4 and there is a reduction in the number of events. Moreover, a third condition has been added, that is, the energy exchanged with the UPG should be less than $2.2 \mathrm{kWh}$. As it can be observed in figure 7 these conditions have provided a group of solutions which comprise $\beta$ values within the interval $[10 \%, 16 \%]$ and $[24 \%, 28 \%]$, and $T_{\max }$ between $[30 \mathrm{~s}, 80 \mathrm{~s}$. Finally, to evaluate the effectiveness of the proposed control architecture, a selection of these solutions has been analyzed and their simulations results have been included within Section 6 .

\section{Results and discussion}

In this section, the effectiveness of the proposed control architecture has been tested by means of simulation tests performed for a typical summer day in Seville under clear-sky conditions. The main goal of these simulations is to evaluate if the proposed event-based state-space MPC control architecture is able to 


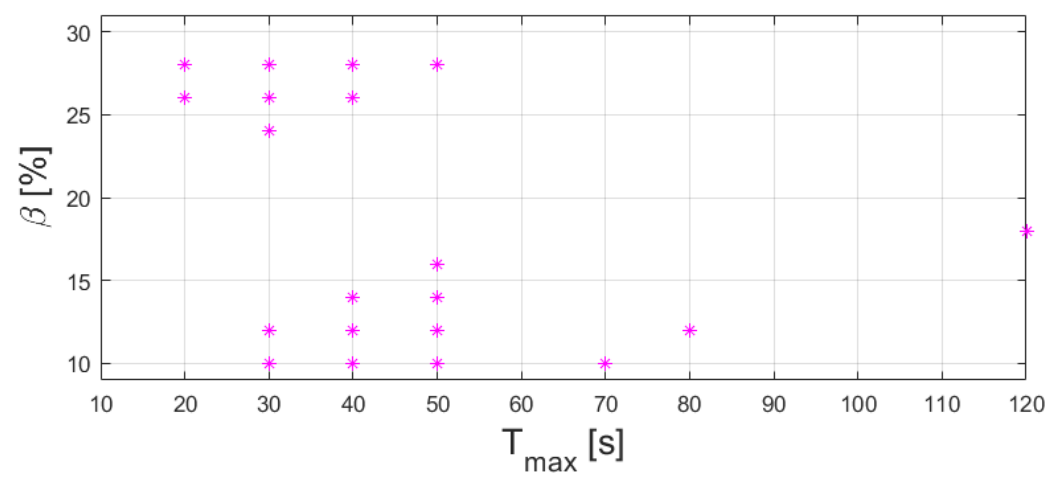

Figure 7: Set of optimal solutions.

satisfy the total energy demanded by the office-room (see figure 3), minimizing the number of actuations without deteriorating significantly the performance of the control system in comparison with a classic state-space MPC controller. A selection of points from Figure 7 has been done.

From the analysis of the graphical results it cannot be established clearly if the event-based state-space MPC architecture is better than the classical one. Hence, an analytical study has been performed taking into consideration some indexes related to set-point tracking, the exchange of energy with the UPG and the computing time. Table 2 shows the results from this analytical study. To analyse the performance of the output variables, that is, SOC and $L O H$, the Integral Square Index (ISE) criterion has been used. It is defined as $I S E_{x}=\int_{0}^{\infty} e^{2}(t) d t$, where $e(t)$ represents the error between the fixed set-point and the output variable. Besides, the amount of energy exchanged with the UPG in $[k W h], E_{\text {grid }}$, has been calculated for each simulation test and for the classic state-space MPC approach. Furthermore, to compare both approaches from a computing time point of view, the total time spent to simulate 24 hours has been obtained for each simulation. Finally, the variables used to select the optimal values for $T_{\max }$ and $\beta$ parameters, that is, the cost function evaluation $J_{\text {eval }}$ and the number of events, have been also included. 

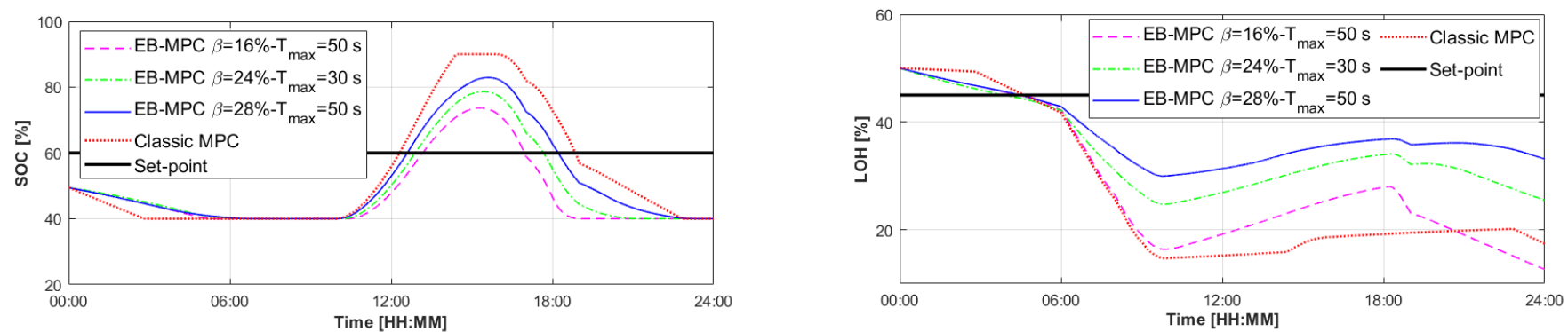

a) SOC and $\mathrm{LOH}$ Set-point tracking
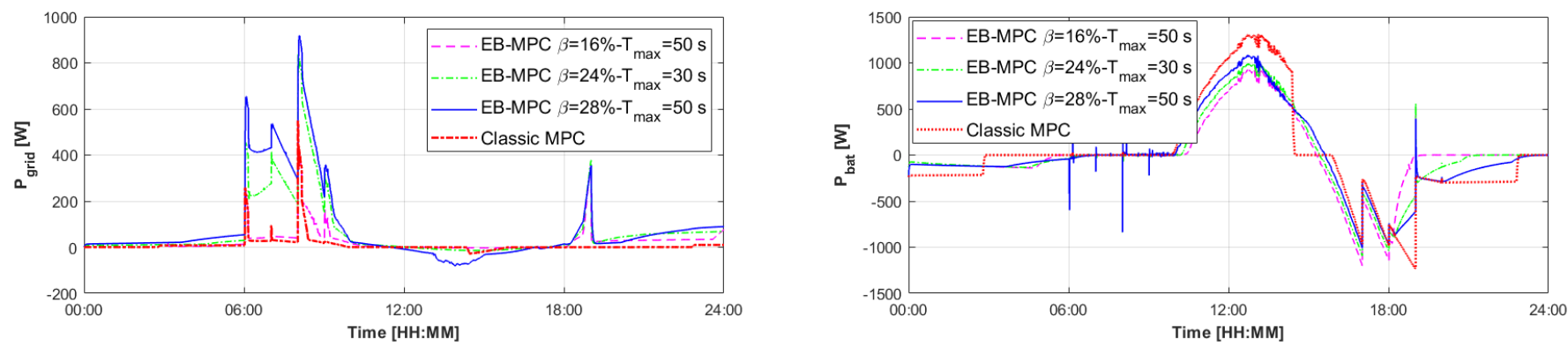

b) Power exchanged with the UPG (left) and the battery bank (right)
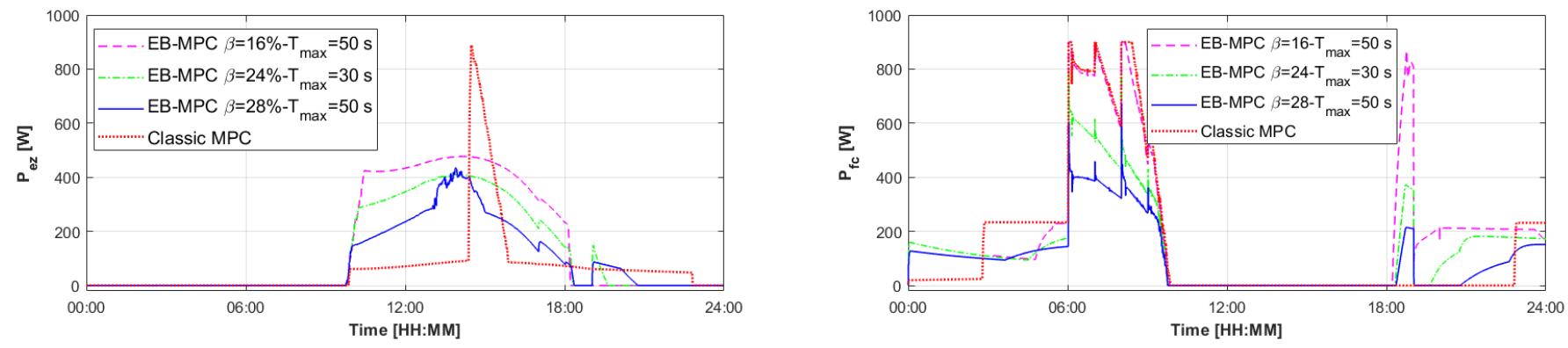

c) Power exchanged with the electrolyzer (left) and the fuel cell (right)

Figure 8: Simulation results for different values of $\beta$ and $T_{\max }$ 
Regarding the performance of the control system, it can be observed that the best result for $J_{\text {eval }}$ index is achieved using the classic state-space MPC. In relation to the results of event-based approach, it can be seen that the number of events is reduced by both increasing $T_{\max }$ or $\beta$ parameters. However, the energy exchanged with the UPG is higher than the classic state-space MPC for all the simulated scenarios. Nevertheless, the performance of the ISE criterion for output variables is better than the one reached with the classic MPC. Therefore, it can be concluded that with the control architecture presented in this paper it is possible to reduce significantly the number of events, and consequently the degradation of the storage devices, at the expense of reducing the performance of the cost function evaluation. Hence, a trade-off solution can be achieved by choosing an event-based strategy with $T_{\max }=30 \mathrm{~s}$ and $\beta=24 \%$. This configuration can also reduce computing time a $70 \%$ approximately without deteriorating the cost function value too much.

Finally, it is necessary to underline that the main objective of developing control strategies for an optimal energy management in a microgrid is not to guarantee set-point tracking of $S O C$ and $L O H$ but to assure that the energy demanded is satisfied by minimizing, at the same time, other matters such as the energy exchanged with the UPG and the degradation of the storage devices. Nevertheless, another important issue that should be taken into consideration is to increase as much as possible the lifetime of the different actuators. In general, the dynamics of a microgrid is very fast, and thus, a sampling period for the classical SSMPC equal to $10 s$ had to be selected, which implies a total of 8640 control actions along one day. Therefore, trying to reduce the number of actuations is very important. According to the simulation results included in this Section, the proposed event-based state-space MPC approach has been able to reduce the number of events around $65 \%$ at the expense of an acceptable deterioration of the cost function value and a little increment in the energy exchanged with the UPG. 


\section{Conclusions}

In this paper, an event-based state-space MPC control architecture has been presented to manage a renewable-energy based microgrid used to provide the energy demanded by an office-room. The main objective of this control approach was to reduce the number of changes in the manipulated variables (avoiding storage devices degradation) maintaining, at the same time, the performance of the microgrid. More in detail, the energy demanded by an office-room has been estimated taking into consideration thermal comfort requirements. To evaluate the goodness of the proposed control approach, simulation results for a typical summer day have been performed under different scenarios. A comparison with a classic state-space MPC control system has been also included. The obtained results are promising and the main conclusion which can be inferred from them is that the control architecture presented in this paper allows the significant reduction of the number of changes in the control signal at the expense of slightly reducing the performance of the cost function evaluation. For instance, in the simulation scenario with $T_{\max }=30 \mathrm{~s}$ and $\beta=24 \%$ a reduction of the number of events equal to $65 \%$ has been reached.

As for future works, the type of events proposed in this paper will be enlarged by simulating a dynamic number of rooms in the system or by including electrical vehicles as a mean to store energy. Moreover, the obligation of satisfying the whole demand will be removed allowing the control architecture to decide the most appropriate time instant to satisfy this demand. Therefore, a demand side management strategy which considers both users' comfort sensation and microgrid operation will be developed and compared with the results presented in this paper.

\section{References}

[1] A. Nuñez-Reyes, D. Marcos, C. Bordons, M. Ridao, Optimal scheduling of grid-connected PV plants with energy storage for integration in the elec- 
tricity market, Solar Energy 144 (2017) 502-516.

[2] D. Olivares, A. Mehrizi-Sani, A. Etemadi, C. Canizares, R. Iravani, M. Kazerani, A. Hajimiragha, O. Gomis-Bellmunt, A. Saeedifard, R. PalmaBehnke, G. Jimenez-Estevez, N. Hatziargyriou, Trends in microgrid control, IEEE Transactions on Smart Grid 5(4) (2014) 1905-1919.

[3] C. Bordons, F. García-Torres, L. Valverde, Optimal management of microgrids with renewable generation (in Spanish), Revista Iberoamericana de Automática e Informática Industrial 12 (2015) 117-132.

[4] W. Gu, Z. Wu, X. Yuan, Microgrid economic optimal operation of the combined heat and power system with renewable energy, Proceedings of the IEEE Power and Energy Society General Meeting, Minneapolis, Minnesota, USA, July 25-29, 2010.

[5] P. Velarde, L. Valverde, J. Maestre, C. Ocampo-Martinez, C. Bordons, On the comparison of stochastic model predictive control strategies applied to a hydrogen-based microgrid, Journal of Power Sources 343 (2017) 161-173.

[6] Z. Wu, Q. Jia, X. Guan, Optimal control of multiroom HVAC system: An event-based approach, IEEE TRANSACTIONS ON CONTROL SYSTEMS TECHNOLOGY, 24 (2) (2016) 662-669.

[7] E. Mojica-Nava, C. Macana, N. Quijano, Dynamic population games for optimal dispatch on hierarchical microgrid control, IEEE Transactions on Systems, Man and Cybernetics: Systems 44(3) (2014) 306-317.

[8] R. Negenborn, M. Houwing, B. Schutter, J. Hellendoorn, Model predictive control for residential energy resources using a mixed-logical dynamic model, Proceedings of the International Conference on Networking, Sensing and Control (ICNSC'09), Okayama, Japan, March 26-29, 2009.

[9] L. Valverde, C. Bordons, F. Rosa, Power management using model predictive control in a hydrogen-based microgrid, Annual Conference on IEEE 
[10] C. Bordons, F. García-Torres, M. Ridao, Model Predictive Control of Microgrids, Springer Nature Switzerland AG, Cham, Switzerland, 2020, ISBN: 978-3-030-24569-6.

[11] H. Fontenot, B. Dong, Modeling and control of building-integrated microgrids for optimal energy management - a review, Applied Energy 254 (2019) 113689.

[12] A. Pawlowski, J. Guzmán, J. Normey-Rico, M. Berenguel, A practical approach for Generalized Predictive Control with an event-based framework, Computers and Chemical Engineering 41 (2012) 52-66.

[13] J. Liu, D. de la Peña, P. Christofides, Distributed model predictive control of nonlinear systems subject to asynchronous and delayed measurements, Automatica 46(1) (2010) 52-61.

[14] D. Bartkevics, Power and control aspects in integrated building management systems with event-based control, Scientific Journal of Riga Technical University 28 (2011) 59-63.

[15] M. Beschi, S. Dormido, J. Sanchez, A. Visioli, L. Yebra, Event-based pi plus feedforward control strategies for a distributed solar collector field, IEEE TRANSACTIONS ON CONTROL SYSTEMS TECHNOLOGY 22(4) (2014) 1615-1622.

[16] A. Pawlowski, I. Fernández, J. Guzmán, M. Berenguel, A. FG, J. NormeyRico, Event-based predictive control of ph in tubular photobioreactors, Computers and Chemical Engineering 65 (2014) 28-39.

[17] L. Valverde, F. Rosa, C. Bordons, Design, planning and management of a hydrogen-based microgrid, IEEE Transactions on Industrial Informatics 9 (3) (2013) 1398-1404. doi:10.1109/TII.2013.2246576. 
[18] F. Garcia-Torres, C. Bordons, Optimal economical schedule of hydrogenbased microgrids with hybrid storage using model predictive control, IEEE

п. Transactions on Industrial Electronics 62 (8) (2015) 5195-5207. doi:10. 1109/TIE.2015.2412524.

[19] M. Castilla, J. Álvarez, F. Rodríguez, B. M, Comfort Control in Buildings, Springer-Verlag, London, England, 2014, ISBN: 978-1-4471-6347-3.

[20] S. Prívara, Z. Váňa, D. Gyalistras, J. Cigler, C. Sagerschnig, M. M, L. Ferkl, Modeling and identification of a large multi-zone office building, Proceedings of the IEEE International Conference on Control Applications, pp. 55-60, Denver, CO, USA, September 28-30, 2011.

[21] M. Trčka, J. Hensen, M. Wetter, Co-simulation of innovative integrated HVAC systems in buildings, Journal of Building Performance Simulation 2 (3) (2009) 209-230.

[22] EnergyPlus, Official web page:, https://energyplus.net/. Accessed July 12, 2018 (2018).

[23] B. C. V. TestBed, Official web page:, https://simulationresearch.lbl. gov/bcvtb. Accessed July 12, 2018 (2018).

[24] Ashrae, International Weather for Energy Calculations (IWEC Weather Files), Users Manual and CD-ROM, Atlanta: ASHRAE, 2001.

[25] ISO7730, Ergonomics of the Thermal Environment: Analytical Determination and Interpretation of Thermal Comfort Using Calculation of the PMV and PPD Indices and Local Thermal Comfort, International Standardization Organization, Geneva, Switzerland, 2005.

[26] D. Rivera, H. Lee, B. MW, H. Mittelmann, Plant-friendly system identification: A challenge for the process industries, Proceedings of the 13th IFAC Symposium on System Identification, pp. 917-922, Netherlands, August 27-29, 2003. 
[27] Ashrae, ASHRAE Handbook - Fundamentals, Refrigerating American Society of Heating and Air-Conditioning Engineers, 2013.

[28] L. Valverde, F. Rosa, A. D. Real, A. Arce, C. Bordons, Modeling, simulation and experimental set-up of a renewable hydrogen-based domestic microgrid, Journal of Hydrogen Energy 38 (27) (2013) 11672-11684. doi:http://dx.doi.org/10.1016/j.ijhydene.2013.06.113.

[29] M. Little, M. Thomson, D. Infield, Electrical integration of renewable energy into stand-alone power supplies incorporating hydrogen storage, International Journal of Hydrogen Energy 32 (10) (2007) 1582 - 1588, eHEC2005. doi:https://doi.org/10.1016/j.ijhydene.2006.10.035.

1 URL http://www.sciencedirect.com/science/article/pii/ S0360319906005209

[30] K. Ȧström, Event Based Control. In: Analysis and Design of Nonlinear Control Systems. In Honor of Alberto Isidori, Springer-Verlag, Berlin Heidelberg New York, 2007, ISBN: 978-3-540-74358-3.

[31] M. Miskowicz, Send-on-delta concept: An event-based data reporting strategy, Sensors 1 (2006) 29-63.

[32] E. Camacho, C. Bordons, Model Predictive Control, Springer-Verlag, London, England, 2007, ISBN: 978-0-85729-398-5.

[33] C. Hans, P. Sopasakis, A. Bemporad, J. Raisch, C. Reincke-Collon, Scenario-based model predictive operation control of islanded microgrids, Proceedings of the 54th IEEE Decision and Control Conference, Osaka, Japan, December 15-18, 2015.

[34] A. Parisio, E. Rikos, L. Glielmo, A model predictive control approach to microgrid operation optimization, IEEE Transactions on Control Systems Technology 22 (5) (2014) 1813-1827.

[35] J. Rossiter, Model based Predictive Control. A Practical Approach, CRC Press, Boca Raton, Florida, 2005, ISBN: 0-8493-1291-4. 
[36] A. Ramírez-Arias, F. Rodríguez, J. Guzmán, M. Berenguel, Multiobjective hierarchical control architecture for greenhouse crop growth, Automatica 48 (2012) 490-498.

[37] G. Liu, J. Yang, J. Whidborne, Multiobjective optimisation and control, Engineering Systems Modelling and Control Series, Recent Studies Press, 2003. 
Table 2: Comparison of selected event-based state-space MPC approach versus classic one.

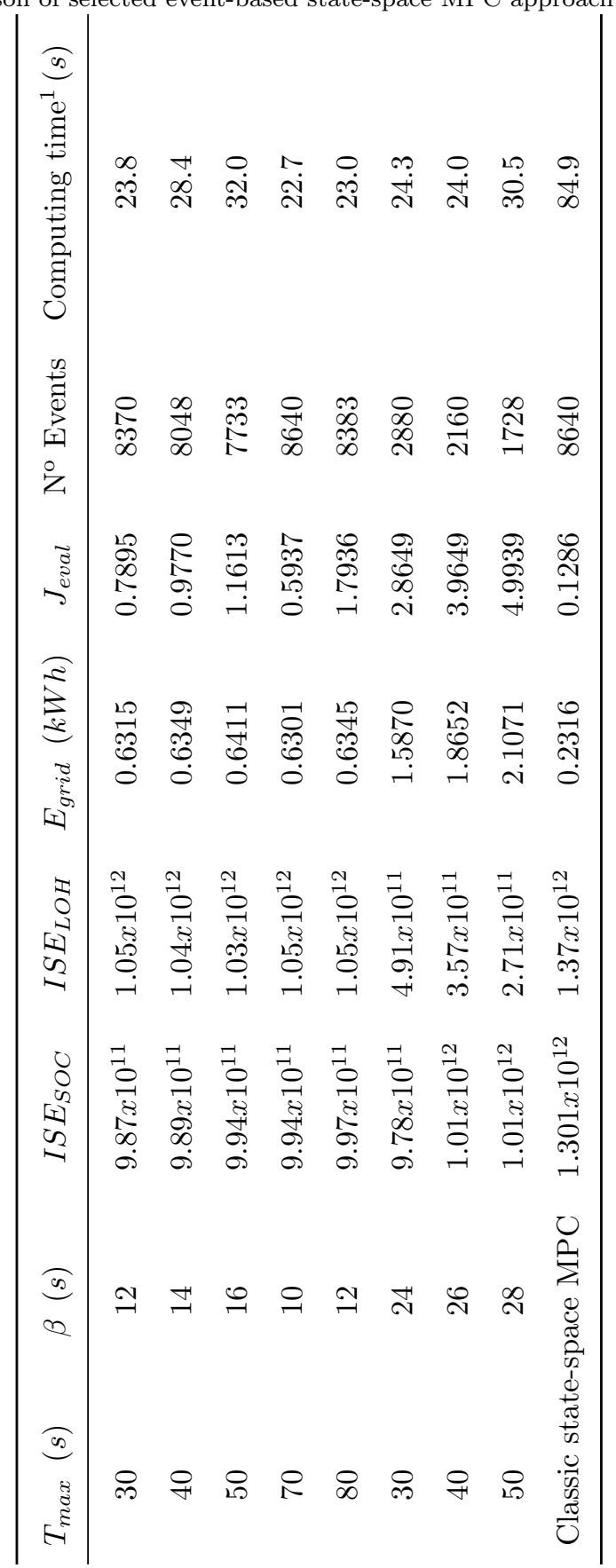

${ }^{1}$ Simulations conducted in Intel(R) Core(TM)i7-8700 CPU 3.20GHz RAM 16 GB, Windows 10x64 and Matlab R2016b 
Table .3: Nomenclature

\begin{tabular}{ll}
\hline Acronym & Meaning \\
\hline BCVTB & Building Control Virtual TestBed \\
$C_{\text {max }}$ & Maximum energy capacity of the battery \\
HVAC & Heating, Ventilation and Air-Conditioning \\
LOH & Level of Hydrogen \\
MPC & Model Predictive Control \\
$\eta$ & Conversion efficiency of each storage unit \\
$P_{b a t}$ & Power exchanged with the battery bank \\
$P_{d e m}$ & Power demanded by the office room \\
PEM & Protom Exchange Membrane \\
$P_{e z}$ & Power to the electrolyzer \\
$P_{f c}$ & Power obtained from the fuel cell \\
$P_{g r i d}$ & Power exchanged with the UPG \\
$P_{H 2}$ & Power applied to the hydrogen path \\
$P_{n e t}$ & Power difference between $P_{r e n}$ and $P_{d e m}$ \\
PLC & Programmable Logic Controller \\
PMV & Predicted Mean Vote \\
PRBS & Pseudo-Random Binary Signal \\
$P_{r e n}$ & Power originated by renewable energy sources \\
SOC & State Of Charge \\
UPG & Utility Power Grid \\
$V_{\text {max }}$ & Volume of $H_{2}$ that can be stored in the tanks \\
ZSF1 &
\end{tabular}

